\title{
Volatile organic compounds and risk of asthma and allergy: a systematic review and meta-analysis of observational and interventional studies
}

\author{
*Ulugbek B Nurmatov', Nara Tagieva², Sean Semple², Graham Devereux², Aziz Sheikh' \\ ${ }^{1}$ Allergy and Respiratory Research Group, Centre for Population Health Sciences, The University of Edinburgh, Medical School, Edinburgh, UK \\ 2 Division of Applied Health Sciences, The University of Aberdeen, School of Medicine and Dentistry, Aberdeen, UK
}

Received 14th October 2012; accepted 30th November 2012; online 1st March 2013

(C) 2013 Primary Care Respiratory Society UK. All rights reserved.

UB Nurmatov et al. Prim Care Respir J 2013; 22(1): PS9-PS15

http://dx.doi.org/10.4104/pcrj.2013.00010

Keywords volatile organic compounds, asthma, allergy, systematic review

\section{Background}

In westernised countries, asthma and related atopic disorders such as eczema and hay fever are now major public health concerns due to their high prevalence, associated significant ill health, and high societal and healthcare costs. ${ }^{1,2}$ There is convincing evidence that the prevalence of asthma and atopic disease has markedly increased in westernised countries since the early 1960s. ${ }^{3-12}$ Such rapid increases in disease prevalence are most likely to be a consequence of changing environmental influences. We describe here a protocol to evaluate systematically the evidence that residential exposure to volatile organic compounds (VOCs) increases the risk of developing asthma/atopic disease and investigate whether exposure to VOCs has adverse effects on those with established asthma/atopic disease.

VOCs are important indoor air pollutants produced by evaporation at room temperature from paints, wood, fabrics, cleaning agents, air fresheners, cosmetics, furnishings, and floor/wall coverings. Indoor VOC levels exceed those outdoors ${ }^{13-15}$ and are further increased by, for example, cigarette smoking and/or if a garage is attached to a house (i.e. petrol combustion, storage of paints, solvents, etc). ${ }^{16}$ Increasing use of VOC-containing products has contributed to increased VOC exposure along with more effective insulation and less external ventilation of modern buildings. Possible health effects of indoor VOC exposure are a cause for concern given that people in general - and children in particular - increasingly spend most of their time indoors. ${ }^{14,17}$ There is a substantial literature reporting associations between increased residential VOC exposure and respiratory symptoms, asthma, atopic disease, and reduced lung function; ${ }^{18-23}$ however, the results are overall inconsistent. ${ }^{24,25}$

Recent studies have also highlighted the potential for maternal
VOC exposure during pregnancy to increase the risk of childhood asthma and atopic disease. ${ }^{26,27}$ Animal studies have shown that VOC exposure, particularly during pregnancy, can influence the immune responses of offspring, increasing Th2 polarisation and their susceptibility to the induction of experimental asthma. ${ }^{28,29}$ In humans, increased Th2 polarisation of neonatal immune responses has been associated with maternal VOC exposure during pregnancy. ${ }^{30}$ Although little evidence exists to suggest that VOCs influence fetal lung growth, embryotoxic effects from exposure to airborne VOCs on murine embryonic stem cells have been reported ${ }^{31}$ and adverse effects on rat embryo/fetal development have been linked to VOC release from their cages. ${ }^{32}$

Several developments have led to a renewed interest in the possible effects of residential VOC exposure on asthma/atopic disease: these include the fact that personalised technology able to quantify individual VOC exposure has been developed and is financially viable for inclusion in studies, ${ }^{33}$ recent longitudinal cohort data suggesting an association between antenatal VOC exposure and childhood asthma/atopic disease, ${ }^{34,35}$ and the availability of new technologies and consumer product standards to reduce residential VOC exposure. ${ }^{36,37}$ Also of relevance is the fact that interventions to reduce VOC exposure are feasible by simple advice (e.g. avoiding redecoration, new furniture, air fresheners, perfumed items, increasing ventilation) ${ }^{16}$ and products can be reformulated to have low VOC content.

There is at present no definitive systematic review of the literature relating residential levels of VOCs to the development of asthma/atopic disease in children and adults and those with established asthma/atopic disease.

\footnotetext{
* Corresponding author: Dr Ulugbek B Nurmatov, Allergy and Respiratory Research Group, Centre for Population Health Sciences, The University of Edinburgh, Medical School, Doorway 3, Teviot Place, Edinburgh EH8 9AG, UK. Tel: +44 (0)131650 2677 E-mail: Ulugbek.Nurmatov@ed.ac.uk
} 


\section{Aims}

We plan to conduct a systematic review and meta-analysis evaluating the relationship between VOC exposure and (1) the development of asthma/atopic disease and (2) in children and adults with established disease, the risk of exacerbations of asthma/atopic disease.

\section{Methods}

\section{Criteria for considering studies for this review} Types of studies

All analytical studies (i.e. cohort, case-control and cross-sectional) and interventional studies (i.e. randomised controlled trials (RCTs), quasi-RCTs, controlled clinical trials (CCTs), controlled before-after and interrupted time series) will be included.

\section{Types of participants}

Studies of subjects relevant to children and adults (i.e. mothers during pregnancy, infants, children and adults) will all be eligible for inclusion.

\section{Types of exposure}

Studies investigating the role of non-occupational (residential, school, day care) exposure to VOC (antenatal, postnatal, early childhood, childhood, adult) in the development and exacerbation of asthma/atopic disease in children and adults will be included.

\section{Types of outcome measures}

- Primary prevention: incidence or prevalence of asthma, eczema, hay fever (the number of new cases, i.e. incidence of asthma, eczema, hay fever; incidence of validated respiratory, dermal, nasal symptoms, lung function, atopic sensitisation).

- Secondary prevention: measures of increased disease activity by any objective measure (lung function, symptom scores, exacerbations, medication usage, healthcare utilisation, quality of life).

\section{Exclusion criteria}

Reviews, letters, editorials, conference abstracts, papers reporting occupational exposure to VOCs, outdoor exposure, estimated/modelled exposure, biomarkers of exposure and those that used surrogate indicators of exposure not objectively quantified (e.g. use of the household chemicals, renovation/painting in the house), and chamber exposure studies are excluded. We have also excluded studies that examined VOCs as tobacco smoke markers and studies on non-specific symptoms (e.g. nasal/throat irritation, Sick Building Syndrome [SBS]).

\section{Research methods for identification of studies}

Electronic searches will include MEDLINE (1966-2012), EMBASE (1980-2012), Cochrane Library (1992-2012), LILACS (1986-2012), ISI Web of Science (1970-2012), BIOSIS (1969-2012), Global Health (1987-2012), AMED (1985-2012), TRIP (2003-2012), CAB (1910-2012), CINAHL (1937-2012). The bibliographies of all eligible studies will be scrutinised to identify additional possible studies. Unpublished and ongoing work and research in progress will be studied by searching key Internet-based databases (www.clinicaltrials.gov; www.controlled-trials.com; www.scholar. google.co.uk). In addition, to extend our search for published, unpublished and ongoing studies, we will contact experts in the field. No language restrictions will be imposed and translations will be sought where necessary. Details of the search strategy are shown in Appendix 1.

\section{Study selection}

Titles and abstracts of trials identified from the searches will be checked by two members of the research team. The full text of all retrieved potentially eligible studies will be independently assessed against the above criteria by two reviewers. The decision on which of the studies fit the inclusion criteria and a record of the methodological quality of eligible studies will be made (see below). Any disagreements will be resolved by discussion between the reviewers or, if necessary, arbitration by a third reviewer.

\section{Assessment of methodological quality}

The assessment and documentation of the methodological quality of included controlled trials will follow the Cochrane approach using the methods detailed in section 8 of the Cochrane Handbook for Systematic Reviews of Interventions. ${ }^{38}$ Intervention studies will be assessed using the Cochrane Effectiveness and Practice Organisation of Care (EPOC) guidelines. The following seven parameters will be used to assess trial quality: random sequence generation; allocation concealment; blinding of participants and personnel; blinding of outcome assessment; incomplete outcome data; selective reporting; and other bias. Each parameter of trial quality will be graded as (A) low risk of bias; $(B)$ moderate risk of bias; or (C) high risk of bias, and an overall assessment for each controlled trial using the same three criteria will be made. Observational studies will be similarly assessed using the Effective Public Health Practice Project (EPHPP) quality assessment tool for quantitative studies. ${ }^{39}$ Reviewers will not be masked to study details. Agreement of reviewers on methodological quality assessment will be assessed and disagreements will be resolved by discussion.

\section{Data extraction}

Two reviewers will independently extract data using customised data extraction forms. For quality assessment of included papers, both reviewers will resolve any disagreements by discussion; in the case of consensus not being reached, a third reviewer will be involved and, if necessary, arbitrate.

\section{Data analysis}

Review Manager 5.1 will be used for data analysis and quantitative data synthesis. For dichotomous data, individual and pooled statistics will be calculated as relative risks (RR) with 95\% confidence intervals $(95 \% \mathrm{Cl})$. For continuous data, individual and pooled statistics will be calculated as mean differences and/or standardised mean differences with $95 \% \mathrm{Cl}$. Consideration will be given to the appropriateness of meta-analysis in the presence of significant clinical or statistical heterogeneity. Heterogeneity will be tested for using the $\mathrm{I}^{2}$ statistic and significant heterogeneity assumed if $\mathrm{I}^{2}$ is greater than $40 \%$ (i.e. more than $40 \%$ of the variability in outcome between trials could not be explained by sampling variation). ${ }^{40}$ Separate meta-analysis using random effects modelling will be undertaken for the main outcomes of interest (i.e. development of asthma/atopic disease and exacerbations of asthma/atopic disease). Subgroup analysis will be performed for various types of VOC exposure and sensitivity analysis will be performed on the basis of 
study quality. Evidence of publication bias will be assessed graphically using funnel plots. ${ }^{41,42}$

\section{Sensitivity analysis}

Sensitivity analysis will be undertaken on the basis of removing studies judged to be at highest risk of bias. Where there is uncertainty, authors will be contacted for clarification or additional information.

\section{Reporting}

The overall reporting of the systematic review will be guided by the PRISMA statement. ${ }^{43}$

\section{Handling editor Mohammed Osman Yusuf}

Acknowledgements We thank Marshall Dozier for her support with constructing the search strategy.

Conflicts of interest The authors declare that they have no conflicts of interest in relation to this protocol.

Contributorship All authors were involved in conceiving and designing this systematic review protocol.

Funding Funding was obtained from the Chief Scientist's Office of the Scottish Government (CSO CZG/2/573).

Protocol registration The protocol is registered with PROSPERO CRD42012003089.

\section{References}

1. Asthma UK. Where do we stand? 2004. Available from: http://www. asmabronquica.com.br/medical/wheredowestand[1].pdf?id=92 (accessed 8 Oct 2012).

2. Asthma UK. Living on a knife edge. 2004. Available from: http://asthma.org.uk/media/94172NAD\%20report.pdf (accessed 8 Oct 20012).

3. Simpson CR, Sheikh A. Trends in the epidemiology of asthma in England: a national study of 333,294 patients. J $R$ Soc Med 2010;103(3):98-106. http://dx.doi.org/10.1258/jrsm.2009.090348

4. Simpson CR, Newton J, Hippisley-Cox J, Sheikh A. Incidence and prevalence of multiple allergic disorders recorded in a national primary care database. $J R$ Soc Med 2008;101(11):558-63. http://dx.doi.org/10.1258/irsm.2008.080196

5. Ghouri N, Hippisley-Cox J, Newton J, Sheikh A. Trends in the epidemiology and prescribing of medication for allergic rhinitis in England. I $R$ Soc Med 2008;101(9):466-72. http://dx.doi.org/10.1258/irsm.2008080096

6. Gupta R, Sheikh A, Strachan DP, Anderson HR. The trends in allergic disorders in the UK. Thorax 2007;62(1):91-6. http://dx.doi.org/10.1136/thx.2004.038844

7. Jenner N, Campbell J, Marks R. Morbidity and cost of atopic eczema in Australia. Australas J Dermatol 2004;45:16-22. http://dx.doi.org/10.1111/j.14400960.2004.00046.x

8. Ninan T, Russell G. Respiratory symptoms and atopy in Aberdeen schoolchildren: evidence from two surveys 25 years apart. BMJ 1992;304:873-5. http://dx.doi.org/10.1136/bmj.304.6831.873

9. Omran M, Russell G. Continuing increase in respiratory symptoms and atopy in Aberdeen schoolchildren. BMJ 1996;312:34. http://dx.doi.org/10.1136/ bmj.312.7022.34

10. Devenny $A$, Wassall $H$, Ninan $T$, et al. Respiratory symptoms and atopy in children in Aberdeen: questionnaire studies of a defined school population repeated over 35 years. BMJ 2004;329:489-90. http://dx.doi.org/10.1136/bmj.38139.666447.F7

11. Tagiyeva N, McNeill G, Russell G, Helms P. Two main subtypes of wheezing illness? Evidence from the 2004 Aberdeen schools asthma survey. Pediatr Allergy Immunol 2008;19:7-12. http://dx.doi.org/10.1111/j.1399-3038.2007.0059.x

12. Mannino DM, Homa DM, Akinbami $\amalg$, et al. Surveillance for asthma: United States, 1980-1999. MMWR Surveill Summ 2002;51:1-13.

13. United States Environmental Protection Agency (EPA). Indoor Air Quality (IAQ). Organic Gases (Volatile Organic Compounds - VOCs). EPA, 2007.

14. Franklin PJ. Indoor air quality and respiratory health of children. Paediatr Respir Rev 2007:8:281-6. http://dx.doi.org/10.1016/j.prrv.2007.08.007.x

15. Zhang J, Smith KR. Indoor air pollution: a global health concern. Br Med Bull 2003;68:209-25. http://dx.doi.org/10.1093/bmb/ldg029
16. Dales R, Raizenne M. Residential exposure to volatile organic compounds and asthma. J Asthma 2004;41:259-70. http://dx.doi.org/10.1081/JAS-120026082

17. Farrow A, Taylor $\mathrm{H}$, Golding J. Time spent in the home by different family members. Environ Technol 1997;8:605-14. http://dx.doi.org/10.1080/09593331808616578

18. Krzyzanowski M, Quackenboss JJ, Lebowitz MD. Chronic respiratory effects of indoor formaldehyde exposure. Environ Res 1990;52:117-25. http://dx.doi.org/ 10.1016/S0013-9351(05)80247-6

19. Rumchev KB, Spickett JT, Bulsara MK, Phillips MR, Stick SM. Domestic exposure to formaldehyde significantly increases the risk of asthma in young children. Eur Respir J 2002:20:403-08. http://dx.doi.org/10.1183/09031936.02.00245002

20. Rumchev, K, Spickett J, Bulsara M, Phillips M, Stick S. Association of domestic exposure to volatile organic compounds with asthma in young children. Thorax 2004;59:746-51. http://dx.doi.org/10.1136/thx.2003.013680

21. Jaakkola JJ, Verkasalo PK, Jaakkola N. Plastic wall materials in the home and respiratory health in young children. Am J Public Health 2000;90:797-9. http://dx.doi.org/10.2105/AJPH.90.5.797

22. Jaakkola JJ, Parise H, Kislitsin V, Lebedeva NI, Spengler JD. Asthma, wheezing, and allergies in Russian schoolchildren in relation to new surface materials in the home. Am J Public Health 2004;94:560-2. http://dx.doi.org/10.2105/AJPH.94.4.560

23. Venn AJ, Cooper M, Antoniak M, Laughlin C, Britton J, Lewis CA. Effects of volatile organic compounds, damp, and other environmental exposures in the home on wheezing illness in children. Thorax 2003;58:955-60. http://dx.doi.org/ 10.1136/thorax.58.11.955

24. Garrett MH, Hooper MA, Hooper BM, Rayment PR, Abramson MJ. Increased risk of allergy in children due to formaldehyde exposure in homes. Allergy 1999;54:330-7. http://dx.doi.org/10.1034/j.1398-9995.1999.00763.x

25. Tavernier G, Fletcher G, Gee I, et al. IPEADAM study: indoor endotoxin exposure, family status, and some housing characteristics in English children. J Allergy Clin Immunol 2006;117:656-62. http://dx.doi.org/10.1016/j.jaci.2005.12.1311

26. Sherriff A, Farrow A, Golding J, Henderson J. Frequent use of chemical household products is associated with persistent wheezing in pre-school age children. Thorax 2005;60(1):45-9. http://dx.doi.org/10.1136/thx.2004.021154

27. Herbarth O, Fritz GJ, Rehwagen M, Richter M, Roder S, Schlink U. Association between indoor renovation activities and eczema in early childhood. Int I Hyg Environ Health 2006;209:241-7. http://dx.doi.org/10.1016/j.jigheh.2006.01.003

28. Bechtel DG, Waldner CL, Wickstrom M. Associations between in utero exposure to airborne emissions from oil and gas production and processing facilities and immune system outcomes in neonatal beef calves. Arch Environ Occup Health 2009;64:59-71. http://dx.doi.org/10.3200/AEOH.64.1.59-71

29. Lim R, Arredouani M, Fedulov A, Kobzik L, Hubeau C. Maternal allergic contact dermatitis causes increased asthma risk in offspring. Respir Res 2007:8:56. http://dx.doi.org/10.1186/1465-9921-8-56

30. Lehmann I, Thoelke A, Rehwagen $\mathrm{M}$, et al. The influence of maternal exposure to volatile organic compounds on the cytokine secretion profile of neonatal T cells. Environ Toxicol 2002;17:203-10. http://dx.doi.org/10.1002/tox.10055

31. Shen $\mathrm{S}$, Yuan $\mathrm{L}$, Zeng $\mathrm{S}$. An effort to test the embryotoxicity of benzene, toluene, xylene, and formaldehyde to murine embryonic stem cells using airborne exposure technique. Inhal Toxicol 2009;21:973-8. http://dx.doi.org/10.1080/ 08958370802687493

32. Lee JC, Ahn TH, Yang YS, et al. Evaluation of embryo-fetal development in rats housed in concrete or hwangto cages during pregnancy. Birth Defects Res $B$ Dev Reprod Toxicol 2008;83:32-9. http://dx.doi.org/10.1002/bdrb.20141

33. Chen C, Campbell KD, Negi I, et al. A new sensor for the assessment of personal exposure to volatile organic compounds. Atmos Environ 2012;54:679-87. http://dx.doi.org/10.1016/j.atmosenv.2012.01.048

34. Diez U, Wetzig H, Schulz R, et al. Effects of indoor painting and smoking on airway symptoms in atopy risk children in the first year of life results of the LARS-study. Leipzig Allergy High-Risk Children Study. Int J Hyg Environ Health 2000;203:23-8. http://dx.doi.org/10.1078/S1438-4639(04)70004-8

35. Sheriff A, Farrow A, Golding J, Henderson J. Frequent use of chemical household products is associated with persistent wheezing in pre-school age children. Thorax 2005;60:45-9. http://dx.doi.org/10.1136/thx.2004.021154

36. Green Seal. Environmental leadership standard for paint and coatings. GS-11. Third edition. 2010. Available from: http://www.greenseal.org/Portals/0/ Documents/Standards/GS-11/gs11_one_pager1.pdf (accessed 8 Oct 2012).

37. Hodgson AT, Shimer DA. Techniques for reducing exposures to volatile organic 
compounds associated with new construction and renovation. 1999. US Department of Energy, Office of Scientific and Technical Information. Available from: http://escholarship.org/uc/item/7576q8sm (accessed 8 Oct 2012).

38. Higgins JPT, Green S. Cochrane Handbook for Systematic Reviews of Interventions. Version 5.1.0. The Cochrane Collaboration, 2011.

39. Effective Public Health Practice Project (EPHPP). Quality assessment tool for quantitative studies. Available from: www.ephpp.ca (accessed 13 Dec 2012).

40. Higgins GP, Thompson SG, Deeks JS, Altman DG. Measuring inconsistency in metaanalysis. BMJ 2003;327(7414):557-60. http://dx.doi.org/10.1136/ bmj.327.7414.557

41. Begg CB, Mazumdar M. Operating characteristics of a rank correlation test for publication bias. Biometrics 1994;50(4):1088-101. http://dx.doi.org/10.2307/ 2533446

42. Egger M, Smith GD, Philips AN. Meta-analysis: principles and procedures. BMJ 1997;315(7121):1533-7. http://dx.doi.org/10.1136/bmj.315.7121.1533

43. Moher D, Liberati A, Tetzlaff J, Altman DG, The PRISMA Group. Preferred Reporting Items for Systematic Reviews and Meta-analyses: The PRISMA statement. PLoS Med 2009;6:e1000097. http://dx.doi.org/10.1371/journal.pmed1000097

\section{Available online at http://www.thepcrj.org}


Appendix 1: Details of search strategy

\section{MEDLINE 1966-present}

1. Exp Hypersensitivity/

2. allerg*.mp.

3. atop*.mp.

4. or/1-3

5. exp Asthma/

6. asthma.mp.

7. Exp Respiratory Tract Diseases/

8. asthmatic children.mp.

9. acute asthmatic attack.mp.

10. night cough*.mp.

11. wheez*.mp.

12. Respiratory hypersensitivity/

13. bronchial disorder.mp.

14. hyper-responsiveness wheez*.mp.

15. Respiratory sounds/

16. Exp Respiration Disorders/

17. Exp Respiratory Function Tests/

18. lung function.mp.

19. ventilatory function. $\mathrm{mp}$.

20. FEV.mp.

21. FEF.mp.

22. FVC.mp.

23. PEF.mp.

24. bronchial hyperreactivity.mp.

25. airway hyperreactivity.mp.

26. bronchial responsiveness.mp.

27. airway responsiveness.mp.

28. or/5-27

29. exp Food hypersensitivity/

30. food allerg*.mp.

31. food hypersensitivity.mp.

32. food hypersensitivities.mp.

33. allergy, food.mp.

34. or/29-33

35. exp Dermatitis, Atopid

36. $\exp$ Eczema/

37. Neurodermatitis/

38. eczema.mp.

39. dermatitis.mp.

40. dermatitides.mp.

41. atopic dermatitis.mp.

42. atopic eczema.mp.

43. eczematous dermatiti*.mp.

44. dermatiti*, eczematous.mp.

45. besnier* prurigo.mp.

46. neurodermatitis.mp.

47. dermatitis, atopic.mp.

48. eczema, atopic.mp.

49. itching.mp.

50. Urticaria/

51. urticaria.mp.
52. or/35-51

53. exp Rhinitis/

54. Rhinitis Allergic Perennial/

55. Rhinitis Allergic Seasonal/

56. hayfever.mp.

57. hay fever.mp.

58. fever, hay.mp.

59. rhiniti*.mp.

60. pollenosis.mp.

61. pollenosis.mp.

62. exp Nasal obstruction/

63. Conjunctivitis/

64. Conjunctivitis, Allergic/

65. conjunctivit*.mp.

66. rhino-conjunctivit*.mp.

67. or/53-66

68. Exp Anaphylaxis/

69. anaphylaxis react*.mp.

70. anaphylactic react*.mp.

71. anaphylactic shock*.mp.

72. anaphylactoid syndrome*.mp.

73. anaphylactoid react*.mp.

74. anaphylactic syndrome*.mp.

75. anaphylactoid shock*.mp.

76. acute systemic allergic react*.mp.

77. idiopathic anaphylaxis.mp.

78. systemic anaphylaxis.mp.

79. or/68-78

80. 3 or 28 or 34 or 52 or 67 or 79

81. analytical stud*.mp.

82. $\exp$ Epidemiologic Studies/

83. $\exp$ Intervention Studies/

84. $\exp$ Evaluation Studies/

85. $\exp$ Comparative Studies/

86. $\operatorname{exp~Follow-up~Studies/~}$

87. $\exp$ Prospective Studies/

88. prospectiv*.mp.

89. $\exp$ Cohort Studies/

90. $\exp$ Case-Control Studies/

91. control.mp.

92. healthy control children.mp.

93. exp Cross-sectional Studies/

94. cohort stud*.mp.

95. cohort.mp.

96. birth cohort.mp.

97. case-control stud*.mp.

98. cross-sectional stud*.mp.

99. etiology.mp.

100. trial.mp.

101. Clinical trial/

102. clinical trial.mp.

103. Controlled Clinical Trial/ 
Appendix 1: Details of search strategy continued

104. controlled clinical trial.mp.

105. Randomized Controlled Trial/

106. Quasi-randomised controlled trial

107. Controlled before and after studies

108. Interrupted time series

109. exp Placebos/

110. exp Random Allocation/

111. exp Double-Blind Method/

112. double-blind design.mp.

113. exp Single-Blind Method/

114. single-blind design.mp.

115. randomised controlled trial.mp.

116. random*.mp.

117. exp Survey/

118. survey.mp.

119. questionnaire*.mp.

120. exp Primary prevention/

121. primary prevention.mp.

122. exp Secondary prevention/

123. secondary prevention.mp.

124. or/81-123

125. exp Ethanol/ or exp Propanols/ or exp Glycols/ or exp butanols/ or exp Heptanol/ or exp Hexanols/ or exp Octanols/ or exp Pentanols/ or exp Octanols/ or exp Aldehydes/ or exp Pyridines/ or exp Amines/ or exp Acetates/ or exp Acetic Acids/ or exp Phthalic Acids/ or exp Formic Acids/ or exp Citric Acid/ or exp Lactic Acid/ or exp Oxalic Acids/ or exp Esters/ or exp Hexanoic Acids/ or exp Trichloroacetic Acid/ or exp Carboxylic Acids/ or exp Isobutyric Acids/ or exp Polyurethanes/ or exp Hydrocarbons/ or exp Ethers/ or exp Ketones/ or exp Dioxins/ or exp Phenols/ or exp cresols/ or exp Hydroquinones/ or exp Azoles/ or exp carbon Disulfide/ or exp Acrylonitrile/ or exp Acetonitriles/ or exp Siloxanes/ or exp Isocyanates/ or exp Anhydrides/ or exp Furans/ or exp Picrates/ or exp Carbon Compounds, inorganic/ or exp Methylmethacrylates/ or exp Morpholines/ or exp Dimethylformamide/ or exp Pyrrolidinones/ or exp Formaldehyde/

126. (ethanol* or propanol* or glycol* or propanediol or butanol* or heptanol* or hexanol* or ethylhexanol or octanol* or octen* or octanon* or pentanol* or butoxyethanol or cellosolve or ethoxyethanol or methoxyethanol or dowanol or butoxydiglycol or butyl carbitol or butyl dioxitol or methylpropanol or isobutyl alcohol or isobutanol or aldehyde* or acetaldehyde* or isobutyraldehyde or isovaleraldehyde or valeraldehyde or formaldehyde or dimethylbenzaldehyde or benzaldehyde* or crotonaldehyde or furfural or hexanal* or hexanaldehyde or pentanal* or acrolein or acrylonitrile or propenal or propionaldehyde or propanal or butanal or butyraldehyde or methylbutanal or heptanal or furaldehyde* or octanal* or benzaldehyde or Decanal or nonanal or pyridine* or aromatic amine* or acetate* or acetic acid* or trichloroacetic acid or monoisobutyrate or diisobutyrate or ester* or dibutyl or formic acid* or hexanoic acid* or caproic acid* or carboxylic acid* or isobutyric acid* or texanol or polyurethan* or polyurethan $\$$ foam or diethyl phthalate or butyl benzyl phthalate or benzyl chloride or chlorotoluene or Octafluorotoluene or tetrahydrofuran or acid anhydride* or isopropanol or isopropyl alcohol or furan* or picric acid or trinitrophenol or isocyanobutan* or carbon monoxide or isobutane or methylpropane or isobutene or isobutylene or methylmethacrylat* or methacrylate* or cyclopropane* or ethanethiol or ethyl mercaptan or ethylene oxide or oxirane or propylene oxide or epoxypropane or dimethylaniline or dimethylacetamide or dimethyl acetamide or bromobenzene or bromochloromethane or bromomethane or chlorodibromomethane or chloromethane or methyl chloride or hydrocarbon* or halocarbon* or aromatic compound* or halogenated organic compound* or alkane* or alkene* or decane* $^{*}$ or dodecane* or undecane* or hendecane* or heptane* or hexane* or nonan* or octan* or tridecane* or pentan* or trimethylpentane or isooctane or methylpentane* or methylhexane or tetradecane or trimethylhexane or hexadecan* or pentadecan* or ethane or dichloroethan* or dutch oil or freon or tetrachloroethan* or tetrachlorethan* or decene or butadiene* or hexachlorobutadiene or lindane).mp. [ $\mathrm{mp}=$ title, abstract, original title, name of substance word, subject heading word, protocol supplementary concept, rare disease supplementary concept, unique identifier]

127. (acetylene or ethyne or chloroprene or isoprene or vinylidene chloride or chloroethan* or chlorethan* or ethyl chloride or dichloroethylen* or dichloroethen* or polyvinyl chloride or PVC or polyvinylchloride or vinyl chloride or chloroethen* or ethylbenzen* or ethyl benzen* or cyclohexanon* or hexanon* or Cycloalkane* or naphthene* or cyclopentan* or methylcyclopentan* or cyclohexan* or cyclohexene* or phenylcyclohexene or methylcyclohexan* or propylcyclohexan* or butylcyclohexan or benzene or benzol* or chlorobenzen* or trichlorobenzen* or dichlorobenzen* or xylen* or methylbenzene* or dimethylbenzen* or styrene* or toluen* or isopropyltoluen* or isopropylbenzene or isopropyl benzen* or propylbenzene or methylcyclopentane or cumene* or cymene or ethyl toluen* or ethyltoluen* or propylbenzen* or trimethylbenzen* or mesitylene or butylbenzene or hexafluorobenzene or perfluorobenzene or phenylcyclohexen* or naphthalene* or naphthalin* or moth balls or napht?ol* or pyrene* or chlorohydrocarbon* or organochlorid* or organochlorin* or chlorocarbon* or chloroalkan* or dichloropropane or dichloropropene or ethylene dibromide or dibromoethane or dibromomethane or methylene bromide or trichloropropane or trichloroethan* or chlorot?ene or methylchloroform or carbon tetrachloride or methane or paraffin or tetrachloromethan* or carbon chloride or tetrachloroethylen* or tetrachlorethylen* or tetrachloroethen* or perchloroethylen* or perchlorethylen* or trichloroethylen* 
Appendix 1: Details of search strategy continued

or trichlorethylen* or trichloroethen* or trichlorethen* or trichlor or dichloromethan* or methylene chloride or trihalomethan* or dibromochloromethane or dibromochloropropane or chloroform or trichloromethan* or bromoform* or bromohydrocarbon* or bromomethan* or bromodichloromethane or terpen* or terpenoid* or camphor or allethrin* or pyrethrin* or pyrethroid* or carene or camphene or limonene or eucalyptol or pinene* or chlorofluorocarbon* or chlorofluorohydrocarbon* or trichlorofluoromethane* or trichloromonofluoromethane or trichlorofluoroethane* or dichlorodifluoromethane or ether* or epichlorohydrin or dioxan* or butoxyethanol* or butoxy ethanol* or ketone* or alkanone* or acetone or propanone or acetophenone* or phenylethanone or butanone* or pentanone or phenol* or carbolic acid or cresol* or hydroxytoluen* or butylhydroxytoluen* or azole* or carbon disulfide or carbon disulphide or carbon bisulfide or siloxan* or heptanon* or ethenylpyridine or butylacetate or hydroquinon* or isocyanate* or diisocyanate* or isopentan* or methylbutan* or fenchon* or terpineol* or thujopsene*).mp. [mp=title, abstract, original title, name of substance word, subject heading word, protocol supplementary concept, rare disease supplementary concept, unique identifier]

128. exp Volatile Organic Compounds/ or exp Volatilization/ or exp Odors/ or Volatile organic compound*.mp. or Volatile organic constituent*.mp. or Volatile organic mixture*.mp. or Volatile chemical*.mp. or volatile compound*.mp. or volatile organic*.mp. or volatile agent*.mp. or Volatile organic chemical*.mp. or volatili?ation.mp. or organic gaseous.mp. or organic gas.mp. or organic gases.mp. or organic aerosol*.mp. or TVOC.mp. or TVOCs.mp. or VOC.mp. or VOCs.mp. or MVOC.mp. or MVOCs.mp. or odo? $\$ 1 . m p$.

129. aerosol.mp. or exp Aerosols/ or exp Deodorants/ or exp Household Products/ or Air freshener*.mp. or exp Aerosol Propellants/ or Cosmetic*mp. or exp Cosmetics/ or exp Construction Materials/ or Building material*.mp. or Building product*.mp. or exp "Facility Design and Construction"/ or exp "Floors and Floorcoverings"/ or Carpet*.mp. or Chipboard.mp. or Chemical based product*.mp. or exp
Disinfectants/ or exp Detergents/ or Cleaning agent*.mp. or Cleaning product*.mp. or Consumer product*.mp. or Decorat*.mp. or exp Disinfection/ or Disinfectant agent*.mp. or redecorat*.mp. or Deodorizer*.mp. or Domestic product*.mp. or Domestic chemical*.mp. or Flooring.mp. or exp Polyurethanes/ or Foam cushion*.mp. or exp "Interior Design and Furnishings"/ or Furnishing*.mp. or Furniture.mp. or Adhesive.mp. or exp Adhesives/ or Glue.mp. or exp Plasticizers/ or exp Household Articles/ or Household Article*.mp. or exp Household Products/ or exp Detergents/ or exp Cosmetics/ or Household Chemical*.mp. or Insect repellent.mp. or exp Insect Repellents/ or Insecticide.mp. or exp Insecticides/ or repellent.mp. or exp Mosquito Control/ or Mosquito coil*.mp. or exp Moths/ or Moth ball*.mp. or Lacquer.mp. or exp Lacquer/ or Solvent.mp. or exp Solvents/ or Surface material*.mp. or exp Solvents/ or exp Paint/ or Paint*.mp. or Perfume.mp. or exp Perfume/ or Plastic*.mp. or renovat*.mp. or exp Plasticizers/ or Plasticizer*.mp. or plasticiser*.mp. or Upholstery.mp. or Varnish*.mp. or Vinyl floor*.mp. or Wax.mp. or exp Waxes/ or exp Wood/ or Wood*.mp. or (freshener spray* or cleaning spray*).mp. or ((gas-phase or gas phase or gaseous-phase or gasphase) adj4 cigarette smoke).mp. or particleboard*.mp. [mp=title, abstract, original title, name of substance word, subject heading word, protocol supplementary concept, rare disease supplementary concept, unique identifier]

130. exp Housing/ or Indoor*.mp. or household*.mp. or exp Air Pollution, Indoor/ or Air Pollutants/ or Air Pollution/ or indoor source*.mp. or residential.mp. or home.mp. or exp Residence Characteristics/ or residence.mp. or school*.mp. or exp Schools/ or domestic.mp. or housing.mp. or exp Ventilation/ or indoor pollutant*.mp.

131. exp Occupational Exposure/ or exp Air Pollutants, Occupational/ or outdoor pollution.mp. or occupational pollution.mp.

132. 125 or 126 or 127 or 128 or 129

133. (130 and 132) not 131

134. 80 and 124 and 133

135. 134 not (animals/not humans/) 